Pacific

Journal of

Mathematics

ENTIRE MEAN CURVATURE FLOWS OF GRAPHS

XIAOLI HAN 


\title{
ENTIRE MEAN CURVATURE FLOWS OF GRAPHS
}

\author{
XiAOLi HAN
}

\begin{abstract}
We consider the evolution of the graph of $f: \mathbb{R}^{n} \rightarrow \mathbb{R}^{n}$ in $\mathbb{R}^{n} \times \mathbb{R}^{n}$ by the mean curvature flow. We prove that the flow exists smoothly for all time if the differential of $f$ has a positive lower bound. Moreover, at each time, the flow remains the graph of a map $f_{t}$.
\end{abstract}

\section{Introduction}

The mean curvature flow deforms the initial surface in the direction of its mean curvature vector. Let $f: \Sigma_{1} \rightarrow \Sigma_{2}$ be a smooth map, and denote the graph of $f$ by $\Sigma$ which is a submanifold of $\Sigma_{1} \times \Sigma_{2}$ by the embedding $F=\mathrm{id} \times f$. Chen, Li, and Tian [2002], Ecker and Huisken [1989], Li and Li [2003], and Wang [2001; 2002] have studied the deformation of $f$ by the mean curvature flow. The key idea of these papers is to consider the quantity $* \omega$, where $\omega$ is the volume form on $\Sigma_{1}$ and $*$ is the Hodge operator with respect to the metric induced on $\Sigma$ by $F$. In fact $* \omega$ is the Jacobian of the projection from $\Sigma$ to $\Sigma_{1}$, and $* \omega>0$ if and only if $\Sigma$ is a graph over $\Sigma_{1}$, by the implicit function theorem. In [Chen et al. 2002; Li and Li 2003; Wang 2001; 2002], the authors independently obtained the results of long time existence and convergence under the condition $* \omega>1 / \sqrt{2}$. We remark that their results depend on the choice of subspace over which $\Sigma$ is written as a graph. In this article, we investigate smooth maps of $\mathbb{R}^{n}$. The advantage of this kind of map is that we can find a good representation of $\Sigma$ via Lewy transformation. This technique is used by Yuan [2002] to prove a Bernstein theorem for special Lagrangian graphs. The difficult is that this case is noncompact. We don't know the behavior of solutions $\Sigma_{t}$ for large time $t$. The surfaces $\Sigma_{t}$ may diverge to $\infty$.

Our result is as follows.

Theorem 1.1. Let $f: \mathbb{R}^{n} \rightarrow \mathbb{R}^{n}$ be a smooth map and $\Sigma$ be the graph of $f$. If $D f \geq \varepsilon$ for some $\varepsilon>0$, then the mean curvature flow of the graph of $f$ remains a graph and exists for all time.

MSC2000: primary 53C44; secondary 53C21.

Keywords: graph, Jacobian of the projection. 


\section{Evolution equation}

First we recall the evolution equation of $* \omega$ along the mean curvature flow. Let $f: \mathbb{R}^{n} \rightarrow \mathbb{R}^{n}$ be a smooth map. The graph of $f$ is an embedded submanifold $\Sigma$ in $\mathbb{R}^{n} \times \mathbb{R}^{n}$ by $F=\mathrm{id} \times f$. Let $\omega$ be the standard volume form on $\mathbb{R}^{n}$. Around any point $p \in \Sigma$, we choose orthonormal frames $\left\{e_{i}\right\}_{i=1, \ldots, n}$ for $T \Sigma$ and $\left\{e_{\alpha}\right\}_{\alpha=n+1, \ldots, 2 n}$ for $N \Sigma$. In the sequel, we use Roman letters $i, j, k, \ldots$ for tangent indices; we use Greek letters $\alpha, \beta, \gamma, \ldots$ for normal indices. We consider the quantity $* \omega$, where $*$ is Hodge operator with respect to the induced metric on $\Sigma$. Then $* \omega=$ $\omega\left(e_{1}, \ldots, e_{n}\right)=\omega\left(\pi_{1}\left(e_{1}\right), \ldots, \pi_{1}\left(e_{n}\right)\right)$, where $\pi_{1}: \mathbb{R}^{n} \times \mathbb{R}^{n} \rightarrow \mathbb{R}^{n}$ is the projection map from $R^{2 n}$ to the tangent space of the domain $\mathbb{R}^{n}$. In fact $* \omega$ is the Jacobian of the projection from $T \Sigma$ to the domain $\mathbb{R}^{n}$. By the singular value decomposition (see [Bretscher 1997]), there exist orthonormal bases $\left\{a_{i}\right\}_{i=1, \ldots, n}$ for the domain $\mathbb{R}^{n}$ and $\left\{a_{\alpha}\right\}_{\alpha=n+1, \ldots, 2 n}$ for the target $\mathbb{R}^{n}$, such that $\lambda_{i \alpha}=\left\langle d f\left(a_{i}\right), a_{\alpha}\right\rangle$ is diagonal. For simplicity, we denote $\lambda_{i(n+i)}=\lambda_{i}$. Now $\left\{e_{i}=\left(1+\lambda_{i \alpha}^{2}\right)^{-1 / 2}\left(a_{i}+\sum_{\alpha} \lambda_{i \alpha} a_{\alpha}\right)\right\}$ forms an orthonormal basis for $T \Sigma$ and $\left\{e_{\alpha}=\left(1+\lambda_{i \alpha}^{2}\right)^{-1 / 2}\left(a_{\alpha}-\sum_{i} \lambda_{i \alpha} a_{i}\right)\right\}$ forms an orthonormal basis for $N \Sigma$. In this setting,

$$
* \omega=\frac{1}{\prod_{i=1}^{n}\left(1+\lambda_{i}^{2}\right)} .
$$

Define the second fundamental form of $\Sigma$ as $h_{i j}^{\alpha}=\left\langle\nabla_{e_{i}} e_{j}, e_{\alpha}\right\rangle$. Recall that $* \omega$ satisfies the equation

$$
\begin{aligned}
& \left(\frac{d}{d t}-\Delta\right) * \omega \\
& \quad=* \omega\left(\sum_{i, j, \alpha}\left(h_{i j}^{\alpha}\right)^{2}-2 \sum_{k, i<j} \lambda_{i} \lambda_{j} h_{i k}^{n+i} h_{j k}^{n+j}+2 \sum_{k, i<j} \lambda_{i} \lambda_{j} h_{i k}^{n+j} h_{j k}^{n+i}\right) ;
\end{aligned}
$$

see [Wang 2002; Chen et al. 2002; Li and Li 2003]. This formula plays the important role in these papers.

\section{Long time existence}

Using Equation (2-1), we now begin to prove our theorem.

Theorem 3.1. If $D f \geq \varepsilon$ for some $\varepsilon>0$, then the mean curvature flow exists for all time.

Proof. Step 1: The key idea is to seek a good representation of $\Sigma$ via Lewy transformation such that $\left|\lambda_{i}\right| \leq 1-\delta$ on the initial surface for some $\delta>0$. This is inspired by Yuan's work [2002]. We rotate the $(x, y) \in \mathbb{R}^{n} \times \mathbb{R}^{n}$ coordinate system to $(\bar{x}, \bar{y})$ by $\pi / 4$, that is, we set $\bar{x}=(x+y) / \sqrt{2}$ and $\bar{y}=(-x+y) / \sqrt{2}$. Then $\Sigma$ 
has a new parametrization

$$
\bar{x}=\frac{1}{\sqrt{2}}(x+f(x)) \quad \text { and } \quad \bar{y}=\frac{1}{\sqrt{2}}(-x+f(x)) .
$$

Since $D f \geq \varepsilon$, we have

$$
\begin{aligned}
\left|\bar{x}^{2}-\bar{x}^{1}\right|^{2} & =\frac{1}{2}\left(\left|x^{2}-x^{1}\right|^{2}+2\left(x^{2}-x^{1}\right) \cdot\left(f\left(x^{2}\right)-f\left(x^{1}\right)\right)+\left|f\left(x^{2}\right)-f\left(x^{1}\right)\right|^{2}\right) \\
& \geq \frac{1}{2}\left|x^{2}-x^{1}\right|^{2} .
\end{aligned}
$$

It follows that $\Sigma$ is still graph over the whole $\bar{x}$ space $\mathbb{R}^{n}$. That means $\Sigma$ has the representation $(\bar{x}, f(\bar{x}))$. Any tangent vector to $\Sigma$ takes the form

$$
\frac{1}{\sqrt{2}}((I+D f(x)) e,(-I+D f(x)) e),
$$

where $e \in \mathbb{R}^{n}$. It follows that

$$
D \bar{f}(\bar{x})=(I+D f(x))^{-1}(-I+D f(x)) .
$$

Noting that $D f \geq \varepsilon$, we have

$$
-I+\delta \leq(D \bar{f}) \leq I-\delta \text { for some } \delta>0 .
$$

For the sake of convenience, we still denote the eigenvalues of $d \bar{f}$ by $\lambda_{i}$. Now we have already shown that $\left|\lambda_{i}\right| \leq 1-\delta$ for some $\delta$. By the theorem in [Tsui and Wang 2004 ] we know that this condition can be preserved along the mean curvature flow. After putting this into Equation (2-1), its right side term in parentheses becomes

$$
\begin{gathered}
\sum_{i, j, \alpha}\left(h_{i j}^{\alpha}\right)^{2}-2 \sum_{k, i<j} \lambda_{i} \lambda_{j} h_{i k}^{n+i} h_{j k}^{n+j}+2 \sum_{k, i<j} \lambda_{i} \lambda_{j} h_{i k}^{n+j} h_{j k}^{n+i} \\
=\delta|A|^{2}+(1-\delta) \sum_{i, k}\left(h_{i k}^{n+i}\right)^{2}+(1-\delta) \sum_{i<j, k}\left(\left(h_{j k}^{n+i}\right)^{2}+\left(h_{i k}^{n+j}\right)^{2}\right) \\
\quad-2 \sum_{k, i<j} \lambda_{i} \lambda_{j} h_{i k}^{n+i} h_{j k}^{n+j}+2 \sum_{k, i<j} \lambda_{i} \lambda_{j} h_{i k}^{n+j} h_{j k}^{n+i} \\
\geq \delta|A|^{2}+(1-\delta) \sum_{i, k}\left(h_{i k}^{n+i}\right)^{2}+(1-\delta) \sum_{i<j, k}\left(\left(h_{j k}^{n+i}\right)^{2}+\left(h_{i k}^{n+j}\right)^{2}\right) \\
-2(1-\delta) \sum_{i<j, k}\left|h_{i k}^{n+i} h_{j k}^{n+j}\right|-2(1-\delta) \sum_{i<j, k}\left|h_{i k}^{n+j} h_{j k}^{n+i}\right| \\
\geq \delta|A|^{2} .
\end{gathered}
$$

Thus we have

$$
\left(\frac{d}{d t}-\Delta\right) * \omega \geq \delta|A|^{2}
$$


According to the maximum principle for parabolic equations, $\min _{\Sigma_{t}} * \omega$ is nondecreasing in time. So $* \omega$ has a positive lower bound, and this implies that $\Sigma_{t}$ remains the graph of a map $f_{t}: \mathbb{R}^{n} \rightarrow \mathbb{R}^{n}$ whenever the flow smoothly exists.

Step 2: The remaining proof is routine. Fix any point $\left(X_{0}, t_{0}\right)$. Then the backward heat kernel $\rho\left(F, X_{0}, t, t_{0}\right)$ at $\left(X_{0}, t_{0}\right)$ is given by

$$
\rho\left(F, X_{0}, t, t_{0}\right)=\frac{1}{\left(4 \pi\left(t_{0}-t\right)\right)^{n / 2}} \exp \left(\frac{-\left|F(x, t)-X_{0}\right|^{2}}{4\left(t_{0}-t\right)}\right) .
$$

First we find a weighted monotonicity formula for $\int_{\Sigma_{t}}(1 / * \omega) \rho\left(F, X_{0}, t, t_{0}\right) d \mu_{t}$ (see [Chen and Li 2004]). Recalling that

$$
\begin{aligned}
\frac{d}{d t} d \mu_{t} & =-|H|^{2} d \mu_{t}, \\
\left(\frac{\partial}{\partial t}+\Delta\right) \rho & =-\left(\left|H+\frac{\left(F-X_{0}\right)^{\perp}}{2\left(t_{0}-t\right)}\right|-|H|^{2}\right),
\end{aligned}
$$

and combining these equations and Equation (3-1), we get

$$
\begin{aligned}
\frac{d}{d t} \int_{\Sigma_{t}} \frac{1}{* \omega} \rho\left(F, X_{0}, t, t_{0}\right) & \\
\leq & \int_{\Sigma_{t}} \phi \rho \Delta \frac{1}{* \omega}-\delta \int_{\Sigma_{t}} \frac{|A|^{2}}{* \omega^{2}} \rho-2 \int_{\Sigma_{t}} \frac{|\nabla * \omega|^{2}}{* \omega^{3}} \rho \\
& -\int_{\Sigma_{t}} \frac{1}{* \omega}\left(\Delta \rho+\left(\left|H+\frac{\left(F-X_{0}\right)^{\perp}}{2\left(t_{0}-t\right)}\right|^{2}-|H|^{2}\right) \rho\right) \\
& -\int_{\Sigma_{t}} \frac{1}{* \omega} \rho|H|^{2} \\
\leq- & \int_{\Sigma_{t}} \rho\left(\frac{2}{* \omega^{3}}|\nabla * \omega|^{2}+\frac{1}{* \omega}\left|H+\frac{\left(F-X_{0}\right)^{\perp}}{2\left(t_{0}-t\right)}\right|^{2}+\delta \frac{|A|^{2}}{* \omega^{2}}\right) \\
& +\int_{\Sigma_{t}}\left(\rho \Delta \frac{1}{* \omega}-\frac{1}{* \omega} \Delta \rho\right) \\
\leq- & \left(\int_{\Sigma_{t}} \frac{1}{* \omega} \rho\left(F, X_{0}, t, t_{0}\right)\left|H+\frac{\left(F-X_{0}\right)^{\perp}}{2\left(t_{0}-t\right)}\right|^{2} d \mu_{t}\right. \\
(3-2) \quad+\delta \int_{\Sigma_{t}} \frac{|A|^{2}}{* \omega^{2}} \rho\left(F, X_{0}, t, t_{0}\right) d \mu_{t} & \left.+\frac{|\nabla * \omega|^{2}}{* \omega^{3}} \rho\left(F, X_{0}, t, t_{0}\right) d \mu_{t}\right) .
\end{aligned}
$$

From this we see that $\lim _{t \rightarrow t_{0}} \int_{\Sigma_{t}}(1 / * \omega) \rho$ exists.

Let $\lambda_{i}$ be positive numbers tending to $\infty$ as $i \rightarrow \infty$ and let $F_{i}$ be the diverging sequence obtained by translating $F$ by $X_{0}$ and then dilating parabolically by $\lambda_{i}$, that is, by taking $(F, t) \rightarrow\left(\lambda_{i}\left(F-X_{0}\right), \lambda_{i}^{2}\left(t-t_{0}\right)\right)$. Denote the new time parameter 
by $s$. Then $t=t_{0}+s / \lambda_{i}^{2}$. Thus,

$$
F_{i}(x, s)=\lambda_{i}\left(F\left(x, t_{0}+\lambda_{i}^{-2} s\right)-X_{0}\right) .
$$

Let $d \mu_{s}^{i}$ denote the induced volume form on $\Sigma_{s}^{i}$ by $F_{i}$. Notice that $* \omega$ is invariant under the parabolic dilation. It is clear that

$$
\int_{\Sigma_{t}} \frac{1}{* \omega} \rho\left(F, X_{0}, t, t_{0}\right) d \mu_{t}=\frac{1}{* \omega} \int_{\Sigma_{s}^{i}} \rho\left(F_{i}, 0, s, 0\right) d \mu_{s}^{i} .
$$

Therefore we get

$$
\begin{aligned}
\frac{d}{d s} \int_{\Sigma_{s}^{i}} \frac{1}{* \omega} \rho\left(F_{i}, 0, s, 0\right) d \mu_{s}^{i} \leq-\left(\int_{\Sigma_{s}^{i}} \frac{1}{* \omega} \rho\left(F_{i}, 0, s, 0\right)\left|H_{i}+\frac{F_{i}^{\perp}}{2\left(t_{0}-t\right)}\right|^{2} d \mu_{s}^{i}\right. \\
+\delta \int_{\Sigma_{s}^{i}} \frac{\left|A_{i}\right|^{2}}{* \omega^{2}} \rho\left(F_{i}, 0, s, 0\right) d \mu_{s}^{i} \\
\left.+2 \int_{\Sigma_{s}^{i}} \frac{|\nabla * \omega|^{2}}{* \omega^{3}} \rho\left(F_{i}, 0, s, 0\right) d \mu_{s}^{i}\right) .
\end{aligned}
$$

Note $t_{0}+\lambda_{i}^{-2} s \rightarrow t_{0}$ for any fixed $s$ as $i \rightarrow \infty$ and $\lim _{t \rightarrow t_{0}} \int_{\Sigma_{t}}(1 / * \omega) \rho d \mu_{t}$ exists. By the above monotonicity formula, this implies that, for any fixed $s_{1}$ and $s_{2}$,

$$
\begin{aligned}
0 \leftarrow \int_{\Sigma_{s_{1}}^{i}} \frac{1}{* \omega} \rho\left(F_{i}, 0, s_{1}, 0\right) d \mu_{s_{1}}^{i} & -\int_{\Sigma_{s_{2}}^{i}} \frac{1}{* \omega} \rho\left(F_{i}, 0, s_{2}, 0\right) d \mu_{s_{2}}^{i} \\
\geq & \int_{s_{1}}^{s_{2}} \int_{\Sigma_{s}^{i}} \frac{1}{* \omega} \rho\left(F_{i}, 0, s, 0\right)\left|H_{i}+\frac{F_{i}^{\perp}}{2\left(t_{0}-t\right)}\right|^{2} d \mu_{s}^{i} \\
& +\delta \int_{s_{1}}^{s_{2}} \int_{\Sigma_{s}^{i}} \frac{\left|A_{i}\right|^{2}}{* \omega^{2}} \rho\left(F_{i}, 0, s, 0\right) d \mu_{s}^{i} \\
& +2 \int_{s_{1}}^{s_{2}} \int_{\Sigma_{s}^{i}} \frac{|\nabla * \omega|^{2}}{* \omega^{3}} \rho\left(F_{i}, 0, s, 0\right) d \mu_{s}^{i} .
\end{aligned}
$$

Since $* \omega$ is bounded below, we have

$$
\int_{s_{1}}^{s_{2}} \int_{\Sigma_{s}^{i}}\left|A_{i}\right|^{2} \rho\left(F_{i}, 0, s, 0\right) \rightarrow 0 \quad \text { as } i \rightarrow \infty .
$$

This implies that for any compact $K \subset R^{2 n}$,

$$
\int_{\Sigma_{s_{i}}^{i} \cap K}\left|A_{i}\right|^{2} \rightarrow 0 \quad \text { as } i \rightarrow \infty
$$

Now we claim that for the graphic mean curvature flow the fact that $* \omega$ has a positive lower bound implies that $\left(X_{0}, t_{0}\right)$ is a regular point. Without loss of 
generality, we assume the origin 0 is on the boundary of $\Sigma_{s_{i}}^{i}$. Since

$$
* \omega=\frac{1}{\prod_{i=1}^{n}\left(1+\lambda_{i}^{2}\right)} \leq \frac{1}{1+\lambda_{i}^{2}}=\frac{1}{1+\left|d f_{t}\right|^{2}}
$$

and because $* \omega$ has a positive lower bound, $\Sigma_{t}$ can be written as the graph of a map $f_{t}: \Sigma_{1} \rightarrow \Sigma_{2}$ with uniformly bounded $\left|d f_{t}\right|$. We consider the diverging sequence of $f$ in $\mathbb{R}^{2 n}$ by $\lambda_{i}$ given by

$$
\tilde{f}_{i}(y)=\lambda_{i} f_{t_{0}+\lambda_{i}^{-2} s_{i}}(y),
$$

where $y \in \mathbb{R}^{n}$. It is clear that $\left|d \tilde{f}_{i}\right|$ is also uniformly bounded and $\lim _{i \rightarrow \infty} \tilde{f}_{i}(0)=0$. By the Arzela theorem, $\tilde{f}_{i} \rightarrow \tilde{f}_{\infty}$ in $C^{\alpha}$ on any compact set. Note that by [Ilmanen 1995, inequality (29)], we have

$$
\left|A_{i}\right| \leq\left|\nabla d \tilde{f}_{i}\right| \leq C\left(1+\left|d \tilde{f}_{i}\right|^{3}\right)\left|A_{i}\right|,
$$

where $\nabla d \tilde{f}_{i}$ is measured with respect to the induced metric on $\Sigma_{s_{i}}^{i}$. From Equation (3-3) we know that

$$
\int_{\Sigma_{s_{i}}^{i} \cap K}\left|\nabla d \tilde{f}_{i}\right|^{2} \rightarrow 0 \quad \text { as } i \rightarrow \infty
$$

which implies that $\tilde{f}_{i} \rightarrow \tilde{f}_{\infty}$ in $C^{\alpha} \cap W_{\text {loc }}^{1,2}$ and that the second derivative of $\tilde{f}_{\infty}$ is 0 . By [Chen and Li 2004, Main Theorem], we know that $\Sigma_{s_{i}}^{i} \rightarrow \Sigma^{\infty}$ and that $\Sigma^{\infty}$ is independent of $s$. Therefore $\Sigma^{\infty}$ is the graph of a linear function. Therefore

$$
\lim _{i \rightarrow \infty} \int \rho\left(F_{i}, 0, s_{i}, 0\right) d \mu_{s_{i}}^{i}=\int \rho\left(F_{\infty}, 0,-1,0\right) d \mu^{\infty}=1
$$

This implies that

$$
\begin{aligned}
\lim _{t \rightarrow t_{0}} \int \rho\left(F, X_{0}, t, t_{0}\right) & =\lim _{i \rightarrow \infty} \int \rho\left(F, X_{0}, t_{0}+\lambda_{i}^{-2} s_{i}, t_{0}\right) \\
& =\lim _{i \rightarrow \infty} \int \rho\left(F_{i}, 0, s_{i}, 0\right) d \mu_{s_{i}}^{i}=1 .
\end{aligned}
$$

White's regularity theorem [2002] tells us that if $\lim _{t \rightarrow t_{0}} \rho\left(F, X_{0}, t, t_{0}\right) \leq 1$, then $\left(X_{0}, t_{0}\right)$ is a regular point. Thus (3-4) tells us that $\left(X_{0}, t_{0}\right)$ is a regular point. We have proved the theorem.

\section{Acknowledgment}

The author thanks ICTP-UNESCO for its hospitality and Professor J. Li for discussions during the work. 


\title{
References
}

[Bretscher 1997] O. Bretscher, Linear algebra with applications, Prentice Hall, Upper Saddle River, NJ, 1997. MR 1429616 Zbl 0896.15001

[Chen and Li 2004] J. Chen and J. Li, "Singularity of mean curvature flow of Lagrangian submanifolds”, Invent. Math. 156:1 (2004), 25-51. MR 2004m:53116 Zbl 1059.53052

[Chen et al. 2002] J. Y. Chen, J. Y. Li, and G. Tian, "Two-dimensional graphs moving by mean curvature flow”, Acta Math. Sin. (Engl. Ser.) 18:2 (2002), 209-224. MR 2003c:53094 Zbl 1028.53069

[Ecker and Huisken 1989] K. Ecker and G. Huisken, "Mean curvature evolution of entire graphs", Ann. of Math. (2) 130:3 (1989), 453-471. MR 91c:53006 Zbl 0696.53036

[Ilmanen 1995] T. Ilmanen, "Singularities of mean curvature flow of surfaces", Preprint, 1995, Available at http://www.math.ethz.ch/ ilmanen/papers/pub.html/sing.dvi.

[Li and Li 2003] J. Li and Y. Li, "Mean curvature flow of graphs in $\Sigma_{1} \times \Sigma_{2}$ ", J. Partial Differential Equations 16:3 (2003), 255-265. MR 2004e:53102 Zbl 1038.53065

[Tsui and Wang 2004] M.-P. Tsui and M.-T. Wang, "Mean curvature flows and isotopy of maps between spheres”, Comm. Pure Appl. Math. 57:8 (2004), 1110-1126. MR 2005b:53110 Zbl 1067. 53056

[Wang 2001] M.-T. Wang, "Deforming area preserving diffeomorphism of surfaces by mean curvature flow”, Math. Res. Lett. 8:5-6 (2001), 651-661. MR 2003f:53122 Zbl 1081.53056

[Wang 2002] M.-T. Wang, "Long-time existence and convergence of graphic mean curvature flow in arbitrary codimension", Invent. Math. 148:3 (2002), 525-543. MR 2003b:53073 Zbl 1039.53072

[White 2002] B. White, "A local regularity theorem for classical mean curvature flow", Preprint, 1999, revised 2002, Available at http://math.stanford.edu/ white/localreg.pdf.

[Yuan 2002] Y. Yuan, "A Bernstein problem for special Lagrangian equations”, Invent. Math. 150:1 (2002), 117-125. MR 2003k:53060 Zbl 1027.53055

Received June 25, 2007. Revised January 21, 2008.

\author{
XiAOLI HAN \\ THE ABDUS SALAm ICTP \\ STRADA COSTIERA 11 \\ 34014 TRIESTE \\ ITALY \\ xhan@ictp.it
}

